\title{
Efectos del consumo de alcohol vía vaginal en adolescentes en cuatro colegios de la ciudad de Medellín, Colombia
}

\author{
Adriana Arango M. ${ }^{1}$, Carla Valentina Vanegas $M .^{a}$ \\ ${ }^{1}$ Ginecóloga, MSc Epidemiología, Universidad CES. Colombia \\ a Alumna, Escuela de Medicina, Universidad CES. Colombia.
}

\begin{abstract}
RESUMEN
Antecedentes: La adolescencia es una etapa de la vida en la que los adolescentes intentan reafirmar su independencia y en muchas ocasiones se puede ver esto rechazando la escala de valores de sus padres. No es de sorprender que en este proceso los adolescentes tengan un consumo mayor de alcohol, el cual puede traer muchas consecuencias tanto en la salud física como psicológica y social. Adicional al consumo vía oral las jóvenes buscan vías alternas para evitar ser descubiertos por la sociedad como es el uso de la vía vaginal. Objetivo: Describir los efectos del uso de alcohol vía vaginal y su entorno social en adolescentes escolarizadas. Método: Estudio descriptivo, en el que se efectuaron 1028 encuestas a las adolescentes de los grados 9, 10 y 11 de cuatro colegios de diferente estrato socio-económico en la ciudad de Medellín. El análisis y la interpretación de los datos se realizaron mediante estadística descriptiva. Resultados: EI consumo de alcohol en las adolescentes escolarizados es frecuente tanto vía oral como vaginal en un $59,2 \%$ y $1,7 \%$ respectivamente. El uso vaginal se acompaña de efectos locales y sistémicos. Los efectos locales en los genitales más frecuentes fueron: ardor, prurito y edema vulvo-vaginal; los efectos sistémicos se presentaron en la primera hora de uso con sensación de "borrachera". Conclusión: El consumo de alcohol vía oral y vaginal es frecuente en las adolescentes con efectos locales y sistémicos.
\end{abstract}

\section{PALABRAS CLAVE: Alcohol, vagina, adolescentes, efectos secundarios}

\section{SUMMARY}

Background: Adolescence is a stage in life when adolescents try to reaffirm their independence. In many occasions this can be observed in their disregard of their parents' values scale. It is not a surprise that through this process adolescents consume more alcohol; which brings many psychological, social and physical health consequences. In addition to the oral consumption, adolescents look for alternative ways to avoid being discovered. Consumption via vagina is one example. Aims: Describe the side effects of alcohol consumption via vagina and the social environment of schooled adolescents. Method: Descriptive study in which 1028 surveys were administered to adolescents from $9^{\circ}, 10^{\circ}$ and $11^{\circ}$ grades in 4 different socio-economic level schools in Medellin. The analysis and interpretation of the data was performed using descriptive statistics. Results: Schooled adolescents frequently consume alcohol orally in $59.2 \%$ as well as via vagina in $1.7 \%$. The consumption via vagina brings with it local and systemic side effects. The most frequent local side effects in the genital area were burning sensation, itching, vulvo-vaginal edema. The only 
systemic side effect present during the first hour after consumption was a drunken sensation. Conclusion: The consumption of alcohol orally and via vagina is frequent among adolescents bringing with it local and systemic side effects.

\section{KEY WORDS: Alcohol, vagina, adolescents, side effects}

\section{INTRODUCCIÓN}

El consumo de alcohol entre los adolescentes según las estadísticas, es cada vez más frecuente $(1,2)$. Sin importar las restricciones legales, ello/ as lo consumen y de alguna forma tienen facilidad para el acceso a las bebidas alcohólicas. En Colombia, según la Organización Panamericana de la Salud, un $76,4 \%$ de los jóvenes consumen alcohol (2). En Antioquia, la Secretaria de Salud entregó cifras de una encuesta hecha a los adolescentes en la que el $39,9 \%$ ha ingerido alcohol, y el $23,2 \%$ lo ha utilizado hasta la embriagues (1).

Debido a las restricciones que tiene la ley frente al consumo de alcohol en menores de edad, además, también debido al rechazo que tiene la sociedad de ver a una mujer con una copa en la mano, las adolescentes crearon la vía vaginal como una alternativa nueva de consumo. No hemos encontrado referencias en la literatura mundial acerca de los efectos del uso de alcohol vía vaginal.

El objetivo de esta comunicación es describir los efectos del uso de alcohol vía vaginal y su entorno social en adolescentes escolarizadas.

\section{SUJETOS Y MÉTODOS}

Se realizó un estudio descriptivo y de corte, con encuesta precodificada con las variables de interés. Dicho formulario fue respondido por 1028 estudiantes de secundaria en cuatro colegios de diferente estrato socioeconómico en la ciudad de MedellínColombia. Se hizo un consentimiento informado en el cual se aseguró la completa confidencialidad de la identidad de cada encuestada.

Las variables recogidas y analizadas fueron: grado de escolaridad, consumo de alcohol vía oral y vía vaginal, motivo del uso vaginal, efectos locales y sistémicos. La muestra fue calculada a criterio de los investigadores a conveniencia por no existir estudios al respecto. El análisis e interpretación de los datos se realizó por medio de estadística descriptiva. A las variables cualitativas se les calculó proporciones. A las variables cuantitativas de les calculó medidas de tendencia central y medidas de dispersión ( $\pm \mathrm{DE})$. Este estudio fue aprobado por el Comité de Ética del Hospital Pablo Tobón Uribe y el Comité de Ética del Parque Explora.

\section{RESULTADOS}

De las 1028 encuestas, se encontró que las jóvenes tenían una edad promedio de 15,9 \pm 1,04 años (rango: 13 - 20 años). La vía de consumo de alcohol más frecuente fue la vía oral, con un $59,2 \%$ (769) mientras que la vía vaginal aportó el 1,7\% $(n=17)$ sin embargo es de anotar que 250 jóvenes nunca habían consumido alcohol (24,3\%). Pero 8 habían utilizado ambas vías. Con relación a la frecuencia de consumo la opción ocasionalmente fue la más referida por las jóvenes con un $52,3 \%$ $(n=538)$ seguido del consumo solo en fines de semana en un $19 \%$ (195) (Tabla I).

Al consumir alcohol por vía oral ellas afirman que fueron inducidas por los amigos en un $42,5 \%$ $(n=552)$ seguido de la voluntad propia en un $11,1 \%$ $(n=144)$ y la familia en un $5,2 \%(n=67)$ (Tabla II).

El consumo de alcohol vía vaginal se encontró en un $1,7 \%(n=17)$ de adolescentes. Dentro de los factores que las inducen a consumir alcohol por vía vaginal tenemos que la curiosidad es el determinante más frecuentemente reportado en un $29,4 \%$, por placer en un $17,6 \%$ siguiendo en frecuencia la presión social y el "estar a la moda" en un $11,7 \%$ cada una (Tabla III).

\section{Tabla I \\ CONSUMO DE ALCOHOL VÍA ORAL EN LAS ADOLESCENTES}

\begin{tabular}{lcc}
\hline Consumo de alcohol vía oral & $\mathrm{n}$ & $\%$ \\
\hline Nunca & 250 & 24,3 \\
Ocasionalmente & 538 & 52,3 \\
Fines de semana & 195 & 19 \\
Frecuentemente & 36 & 3,5 \\
Sin dato & 9 & 0,9 \\
\hline
\end{tabular}




\section{Tabla II \\ INDUCCIÓN AL CONSUMO DE ALCOHOL VÍA ORAL}

\begin{tabular}{lcc}
\hline Inducción al consumo & $\mathrm{n}$ & $\%$ \\
\hline Amigos & 552 & 42,5 \\
Voluntad propia & 144 & 11,1 \\
Familia & 67 & 5,2 \\
Amigos y novio & 29 & 2,2 \\
Mixto & 37 & 3 \\
\hline
\end{tabular}

\section{Tabla III \\ FACTORES QUE LAS INDUCEN A CONSUMIR ALCOHOL VÍA VAGINAL}

\begin{tabular}{llc}
\hline $\begin{array}{l}\text { Factores que las inducen a } \\
\text { consumir alcohol vía vaginal }\end{array}$ & $\mathrm{n}$ & $\%$ \\
\hline Curiosidad & 5 & 29,4 \\
Placer & 3 & 17,6 \\
Presión social & 2 & 11,7 \\
Estar a la moda & 2 & 11,7 \\
Estar a la moda y curiosidad & 2 & 11,7 \\
Placer y curiosidad & 2 & 11,7 \\
Estar a la moda, curiosidad y & 1 & 5,8 \\
placer & & \\
\hline
\end{tabular}

Las formas más frecuentes de preparar el alcohol antes de aplicarlo en la vagina fueron impregnando un tampón, una toalla o papel higiénico con licor en un $52,94 \%(n=9), 29,41 \%(n=5)$ y $5,88 \%$ $(n=1)$, respectivamente, 2 no responden. Al momento de la aplicación del tampón sienten ardor vaginal en un $41,17 \%(n=7)$ seguido de haber sentido rasquiña vaginal y ardor vaginal en un $23,52 \%$ $(n=4)$ y placer en un $17,64 \%(n=3)$ (Figura 1$)$.

Las adolescentes dicen que sienten mareo o "borrachera" de manera inmediata después de consumir alcohol vía vaginal en un $41,17 \%(n=7)$, entre 30 a 59 minutos en un $35,29 \%(n=6)$ y solo el $5,88 \%(n=1)$ tuvo esa sensación entre 1 y 3 horas más tarde. El efecto persiste más de 2 horas en un $76,47 \%(n=13)$, seguido de un $11,76 \%(n=2)$ entre 1 a 2 horas, un $5,88 \%(n=1)$ menos de 30 minutos, y $5.88 \%(n=1)$ no respondió.
También se les preguntaron los síntomas posteriores al consumo de alcohol vía vaginal que presentaban en la vagina y región genital; el de mayor frecuencia fue el prurito vaginal con un $41,17 \%$ $(n=7)$, ardor vaginal $17,64 \%(n=3)$ y $5,88 \%(n=1)$ sequedad vaginal. El $35,31 \%(n=6)$ restante, no referenció síntomas posteriores al consumo de alcohol vía vaginal.

Se les preguntó por síntomas propios de la resaca, y el de mayor frecuencia fue cefalea con un $70,58 \%(n=12)$ seguido de pereza, desgano y sed con un $5,88 \%(n=1)$.

Con relación a la vida sexual, un $70,58 \%(n=12)$ de las adolescentes consumidoras de alcohol vía vaginal tuvo relaciones sexuales posteriores al consumo. Los síntomas que ellas presentaron en la vagina después de haber tenido relaciones sexuales fueron en un $23,52 \%(n=4)$ dolor, ardor y rasquiña en la vagina, seguido de un $5,88 \%(n=1)$ que dijo haber sentido sensación de hinchazón.

Los motivos principales para la administración vaginal fueron la ausencia de aliento a alcohol con un $64,70 \%(n=11)$, el no llegar a la embriaguez extrema con un $11,76 \%(n=2)$, y el no ser descubiertas por sus padres con un $5,88 \%(n=1)$ (Tabla IV).

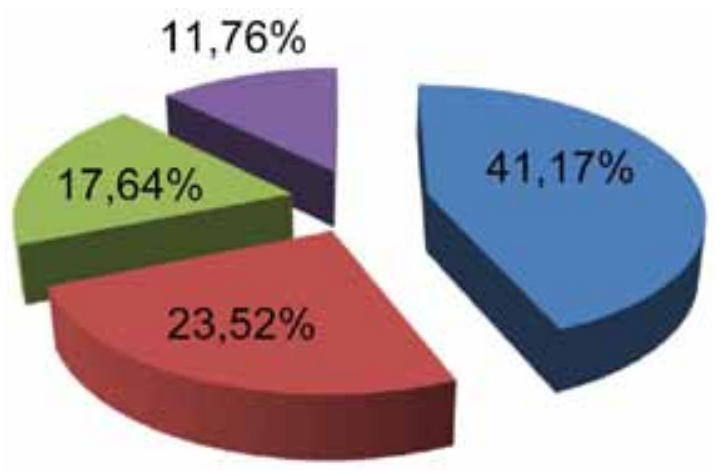

= Ardor vaginal

\section{- Rasquiña y ardor vaginal = Placer \\ * Sensación de hinchazón}

Figura 1. Síntomas y sensaciones al consumir alcohol vía vaginal. 


\section{Tabla IV \\ MOTIVOS PARA EL CONSUMO DE ALCOHOL VÍA VAGINAL}

\begin{tabular}{lcc}
\hline Motivos & $\mathrm{n}$ & $\%$ \\
\hline Para no tener aliento a licor & 11 & 64,7 \\
$\begin{array}{l}\text { Para no alcanzar un alto grado } \\
\text { de embriaguez }\end{array}$ & 2 & 11,76 \\
$\begin{array}{l}\text { Aliento a licor y engañar a los } \\
\text { padres }\end{array}$ & 3 & 17,6 \\
Para engañar a mis padres & 1 & 5,88
\end{tabular}

\section{DISCUSIÓN}

El alcohol es una droga que ya está muy integrada en nuestra sociedad, por lo tanto el consumo de este no genera rechazo social; sin embargo, adicional a los efectos en el estado mental y físico, existe un poco de rechazo frente al consumo de alcohol por parte de la mujer.

Los adolescentes que consumen alcohol niegan sufrir alcoholismo solo porque entre semana no lo consumen, y solo lo hacen los fines de semana, pero el problema es que cuando lo consumen pasan los límites y esto puede causar problemas a futuro por fármaco dependencia (3).

Los adolescentes consumen alcohol para sentirse bien, para pertenecer a un grupo social, por el gusto hacia su sabor y desinhibirse, y así quitarse vergüenzas para hacer cosas que en otro estado no harían (3); esto confirma uno de los puntos de los resultados de la encuesta, en que las adolescentes encuestadas consumen alcohol para sentirse bien y para pertenecer a un grupo social (4).

La edad del inicio del consumo de alcohol cada vez es más precoz, según los datos de la encuesta sobre drogas a la población escolar 1998 (Delegación del Gobierno para el Plan Nacional sobre Drogas) es de 13,6 años, y la edad media de inicio de consumo semanal se sitúa en los 14,9 años, esta fuente también afirma que las chicas registran mayor prevalencia de consumo de alcohol, aunque en cantidades menores (5); en el presente estudio la edad promedio fue mayor (15,9 años).

Los resultados que arrojaron las encuestas frente al uso de alcohol vía oral, son de un $59,2 \%$ de la población encuestada, mientras que en Antioquia, las estadísticas indican que es un $39,9 \%$ de adolescentes que consume alcohol (1).

Debido a que no existe información en la literatura acerca del consumo de alcohol vía vaginal, la única información que ahora se tiene es el presente estudio, el cual dio como resultados que un $1,7 \%$ $(17 / 1028)$ de las adolescentes encuestadas consume alcohol por vía vaginal, y el mecanismo principal es la impregnación del tampón y la causa de su uso fue curiosidad principalmente, con efectos rápidos como la sensación de mareo o borrachera y locales como prurito y ardor vulvovaginal.

Todos estos resultados se pueden relacionar con varias enfermedades asociadas al sistema reproductor femenino, como lo son la vaginitis por cándida, vaginosis bacteriana, enfermedad pélvica inflamatoria, entre otras (5-7).

Es necesario poner atención a este problema, ya que es deseable evitar el consumo de alcohol en adolescentes, y las consecuencias locales, sistémicas y mentales derivadas de su uso como es la enfermedad alcohólica $(8,9)$. Aunque es importante poner atención al alcoholismo hay que mirar las costumbres relacionadas a éste, ya que el consumo de alcohol también puede llevar al consumo de drogas, lo cual también se quiere evitar y tenido en cuenta (10).

\section{CONCLUSIÓN}

El consumo de alcohol vía oral y vaginal es frecuente en las adolescentes con efectos locales y sistémicos.

\section{REFERENCIAS}

1. Las drogas ganan terreno. Rojas D. Acceso: 18 de Junio de 2010. Hallado en: http://www.elmundo.com/ portal/resultados/detalles/?idx=151722.

2. Alcohol y Salud pública en las Américas, un caso para la acción. Organización Panamericana de la Salud. Hallado en: http://www.paho.org/Spanish/DD/PIN/ A\&SP.pdf.

3. Alcoholismo en la adolescencia, Adicción al alcoholismo. Centro de Psicología Bilbao. Hallado en: http:// www.centro-psicologia.com/es/alcoholismo-adolescentes.html

4. Adicción al alcohol y otras drogas en adolescentes. Psicólogos sur Chiclana. Hallado en: http://www. psicologos-sur.com/es/adiccion-al-alcohol-y-drogas. $\mathrm{html}$.

5. El alcohol en los adolescentes. En: Contribuciones a las Ciencias Sociales. Ferrer Lacosta, C. Hallado en: www.eumed.net/rev/cccss/03/cfl3.htm

6. Al-Sanouri I, Dikin M, Ayman O. Soubani. Critical care aspects of alcohol abuse. South Med J 2005;98:37281.

7. Akhter S, Beckmann K, Gorelick M. Update on sexually transmitted infections. Pediatr Emerg Care 2009;25:608-18.

8. Alcoholismo y adolescencia, tendencias actuales. Rodríguez Sánchez I, Castillo Ledo I, Torres Lugo DJ, 
Jiménez Hernández Y, Zurita Pacheco DM. P62. Hallado en: http://www.paidopsiquiatria.com/numero8/ art5.pdf

9. Bardiera R, Hariyo M, Jaquenod DE Giusti C, Nápoli $\mathrm{H}$. Alcoholismo en los adolescentes ¿Una enfermedad o un entretenimiento? Hallado en: http://www. monografias.com/trabajos13/infalcoh/infalcoh.shtml.
10. Valderrama Zurián JC. Consumo de alcohol. Hallado en: http://www.saludalia.com/Saludalia/web_saludalia/vivir_sano/doc/alcohol_y_drogas/doc/alcohol_jovenes.htm 